Felix D. Ayahao, MD

Department of Ear Nose Throat - Head Neck Surgery Baguio General Hospital and Medical Center
Correspondence: Dr. Felix D. Ayahao Department of ENT-HNS

Baguio General Hospital and Medical Center

Governor Pack Road, Baguio City, Benguet 2600

Philippines

Telefax: (074) 4444176

Reprints will not be available from the author.

The author declared that this represents original materia that is not being considered for publication or has not been published or accepted for publication elsewhere, in full or in part, in print or electronic media; that the manuscript has been read and approved by the author, that the requirements for authorship have been met by the author, and that the author believes that the manuscript represents honest work.

Disclosures: The author signed disclosures that there are no financial or other (including personal) relationships, intellectual passion, political or religious beliefs, and institutional affiliations that might lead to a conflict of interest.

\section{Feeding and Draining Vessel Ligation with Sclerotherapy of High Flow Arteriovenous Malformations in the Head and Neck}

High flow arteriovenous malformations (AVMs) are infiltrative, invading tissue planes and structures and may be life threatening when they bleed. ${ }^{1}$ They have a feeding artery and an anomalous capillary bed shunting blood from the arterial system to the venous system.' The present trend of management of small AVMs is surgical excision with a high success rate. The problematic cases are diffuse AVMs infiltrating structures that render them impossible to totally extirpate surgically without causing much blood loss and tissue damage. The mainstay of management is embolization, surgical resection and reconstruction. ${ }^{2}$ Ligation or proximal embolization (alone) of feeding vessels should never be done because such maneuvers result in rapid recruitment of new vessels from adjacent arteries to supply the AVM nidus. ${ }^{2}$ Incomplete surgical excision definitely leads to recurrences making this type of AVM very difficult to manage.

What is the point of this paper? The complete destruction of the "nidus" of the AVM from the artery to the capillary to the venous component is the only potential cure. ${ }^{3}$ Well and good if there could be a way of doing this by sclerosing the entire vascular malformation. But since sclerosis only works well in low flow vascular malformations and tumors like hemangiomas and poorly or not at all in high flow lesions, ${ }^{4}$ we have to convert this high flow AVM into a "no-flow" or "low flow" AVM by ligating the feeding and draining vessel and injecting the sclerosant intra-arterially thereafter at a dose sufficient enough to blanch out the AVM even up to its peripheral branches. This paper aims to demonstrate how we do this.

\section{DEFINITION OF TERMS}

Vascular malformation: They are a result of abnormal development of vascular elements during embryonic or fetal stages of life. ${ }^{2}$ They originate from mesenchymal cells at an early stage of embryogenesis ${ }^{3}$ and most are present at birth but there are several case reports of these lesions presenting after trauma in adults.' Some AVMs appear as part of a familial genetic disorder called angiomatous syndrome i.e. Rendu-Osler-Weber Syndrome presenting with telangiectasia of the skin and mucous membranes. ${ }^{3}$ Some propose that a defect in vascular stabilization like TGFbeta signaling could be a cause of AVM development. ${ }^{5,6}$ Still, progesterone receptors have been isolated in AVMs explaining their expansion during puberty. ${ }^{7}$

Hemangioma: These are vascular tumors that exhibit endothelial proliferation. ${ }^{2}$ A hemangioma of infancy usually undergoes three stages: a proliferative phase of rapid growth up to 10 to 12 months of age; an involuting phase where growth slows down and signs of regression appear usually at 1 to 7 years; and an involuted phase. ${ }^{2}$

Sclerosants: Agents used in sclerotherapy that induce a toxic effect on the vascular endothelium and results in fibrosis. There are three types: Detergents that disrupt cell membranes by protein theft desaturation i.e. ethanolamine oleate, sodium morrhuate, polidocanol, sodium tetradecyl sulfate; Osmotic agents i.e. sclerodex; and Chemical irritants that damage cell walls by direct contact i.e. chromatin glycerine, polyiodinated iodine. ${ }^{8}$

Sclerodex: an osmotic sclerosant that is a combination of dextrose monohydrate $250 \mathrm{mg} /$ $\mathrm{ml}$ and sodium chloride $100 \mathrm{mg} / \mathrm{ml}$. It shifts water balance through cellular gradient (osmotic) dehydration that leads to endothelial destruction. Since component materials are naturally occurring bodily, it has no molecular toxicity in calibrated dosages. If extravasated, it could cause tissue necrosis. ${ }^{8}$ It is manufactured by OMEGA Laboratories, Ltd. Montreal, QC, Canada. 


\section{REVIEW OF PRESENT PRACTICE AND LITERATURE}

The first task of the physician is to establish a diagnosis whether the lesion is a vascular malformation or a vascular tumor. Taking the history of the patient could point to a diagnosis as vascular tumors like hemangiomas usually proliferate and involute from the time of infancy to about the age of 10. Vascular malformations grow as the child grows and do not involute. Vascular malformations have an arterial supply and a venous drainage and are classified into high or low flow. Capillary, venous and lymphatic types are low flow while arteriovenous malformations are usually high flow.

A high flow AVM has an arterial blood supply and a venous drainage. In rare instances, a vascular malformation could co-exist with a hemangioma forming a mass effect. ${ }^{2}$ On physical examination, a bruit and a strong pulsation (thrill) is appreciated.

The head and neck is the most common location of AVMs at 70\%. When fully developed, they are deeper in color with increasing erythema, local warmth, palpable mass and a bruit. ${ }^{9}$ These malformations are composed of vascular channels lined by flat mature epithelium and are not hypercellular and not proliferative. ${ }^{10}$

Schobinger proposed a staging system for Head and Neck AVM. Stage 1 are AVMs that are quiescent and remain stable for long periods of time. Stage 2 is a time for expansion followed by pain and bleeding. Stage 3 is heralded by destruction of adjacent tissues and ulceration. Stage 4 is presented by decompensation where symptoms of cardiac failure are present. ${ }^{11}$

Ultrasound with color Doppler imaging, Magnetic Resonance Imaging and Phlebography (arteriography/venography) contribute to diagnosis, classification and management. ${ }^{3}$ In our setting were we do not have the facilities, we use CT- angiography. These imaging modalities should be used to evaluate the characteristics of the lesion, such as size, flow velocity, flow direction, relation to surrounding structures and lesion content. ${ }^{3}$ Ultrasound demonstrates flow rates, contrast-enhanced magnetic resonance imaging (MRI) shows presence or absence of a mass and CT angiography reveals the arborization (the blood supply and drainage) of the vascular anomaly.

Vascular tumors like hemangioma, if located in non-strategic areas where function is not impaired can be observed over its developmental phases until involution at about 10 years old. ${ }^{2}$ For hemangiomas that impair function or are possibly life threatening because of potential hemorrhage, these tumors are treated with the following modalities: 1) Intralesional corticosteroids i.e. triamcinolone; 2) Systemic corticosteroids in a tapered dose like prednisolone and some second choice pharmacotherapeutics like interferon, vincristine; and 3) Propranolol. Surgery is indicated in ulcerating, bleeding and life threatening lesions like airway obstruction.?

Over $90 \%$ demonstrate dramatic reduction in size of hemangioma in one to two weeks from the above medical therapeutic modalities. Propranolol has been successfully used as hemangioma treatment since 2008 and is believed to have an antiproliferative effect on the vascular endothelium. The mechanism of action may involve the regulation of growth factors.'

Low flow vascular malformations are treated with sclerotherapy or surgical excision for accessible tumors. These malformations do not regress like hemangioma but grow in time. High flow AVMs are treated with surgical excision if they have limited extent and are surgically accessible. Embolization before surgery decreases bleeding and is the standard. Embolization followed by repeated sclerotherapy is recommended for surgically inaccessible areas. ${ }^{4}$ There is a 64 to $96 \%$ response rate, defined as improvement in symptoms or a reduction in the lesion size after ethanol sclerotherapy of venous low flow malformations. ${ }^{3}$ Partial surgical excision leads to only temporary improvement followed by re-expansion of tumor overtime. ${ }^{9}$ Sometimes, complete resection is not possible in diffuse or infiltrating AVMs and surgery can result in severe disfigurement and impairment of function of involved structures. ${ }^{9}$

\section{METHODS}

After establishing the diagnosis of a high flow AVM with identification of an arterial feeding vessel and a venous draining vessel, surgery is commenced away from the malformation to expose the arterial and venous supplies. The procedure is done under general anesthesia because sclerotherapy of large malformations and vascular tumors is very painful. We do this because we have no interventional radiology services in our hospital. We ligate the feeding artery and if possible, the draining vein to convert the AVM into a "low or no flow" and to allow ample contact time between the sclerosant (sclerogen) and the vessel endothelium. The sclerosant is then injected intravascularly distal to the ligation until all visible malformation blanches out. Aspirating the blood content of the ligated (arterial supply and venous drainage) malformation before introduction of the sclerosant will further potentiate the action of the sclerosant. While injecting the sclerosant slowly, the patient's vital signs are monitored. A drop in the pulse rate is a signal to stop or slow down the injection of the sclerosant because it may be a sign that some sclerosant is escaping the venous drainage and reaching the general circulation in a concentration picked up by the sensors of the vascular system. Injections resume in a slower manner as the vital signs revert to normal. Our sclerosing agent sclerogen is an osmotic agent composed of sodium chloride and dextrose which are naturally present in our body so they are not toxic in manageable concentrations. Other sclerosants can be nephrotoxic so we must be very careful in injecting not to overload the vascular system. The objective is to push the sclerosant to all branches of the malformation to eliminate all possible nidus. The end point of injection is when all cutaneous or mucosal components of the malformation blanch out.

Injection of the sclerosant intravascularly is done under direct visualization to prevent extravascular introduction. A review of literature has this to say about sclerotherapy. Extravascular injection of the sclerosant causes tissue necrosis. ${ }^{4}$ Ethanol injection to high flow fistulous lesions is contraindicated because of high risk of "early wash" into the systemic circulation. ${ }^{4}$ Sclerosants could cause hemolysis, denaturation of blood proteins, thrombus formation and nephrotoxicity. ${ }^{34,12}$ Ethanolamine oleate, in comparison to ethanol, has less effect in the deep vascular layer and no penetrative effect. It is not associated with neuronal side effects despite of the proximity of the nervous system to the vascular system. ${ }^{3}$ 
PRACTICE PEARLS

\section{CASES}

Our first case was a 62-year-old woman with a pinna and periauricular vascular malformation, noted since five years prior to consultation. The inferior concha was bulging and pulsating with a strong bruit. The periauricular area was elevated with microvascular malformations in reddish discoloration. The left posterior auricular artery was identified as the feeding artery and the diagnosis was a high flow AVM. She consulted a hospital in Sacramento, California where she worked and was advised to have a resection of her left ear. She got frightened and decided to come home to the Philippines for a second opinion.

I suggested our procedure which she gladly accepted but warned her of possible pinna necrosis. At least, she said, it is just a possibility and not an outright pinna loss. I dissected $1 \mathrm{~cm}$ below the malformation avoiding any of its extensions below the pinna and mandibular angle and moved towards the external carotid. I immediately located the pulsating, abnormally dilated posterior auricular artery feeding vessel and ligated it. Further dissection deep towards the styloid process revealed the venous drainage that penetrated the mastoid bone toward the direction of the sigmoid sinus. I, too, did the venous drainage ligation. I injected sclerogen distal to the posterior artery ligation after aspiration of $8 \mathrm{cc}$ of AVM blood until all the malformation main mass and the peripheral branches blanched out. Total volume of sclerosant was $10 \mathrm{cc}$.

There was no change in the vital signs as I slowly introduced the sclerosant. I closed the surgical defect and observed the patient for three days in the hospital. There was postoperative pain and swelling in the sclerosed malformation relieved by ice packs and celecoxib $200 \mathrm{mg}$ every 12 hours. After three days, the swelling started to subside and the pain lessened so the patient was sent home. She followed up in a week and the malformation had shrunk. Sutures were removed. Two weeks postoperatively, the malformation was just a trace skin discoloration with no tissue necrosis, no more bulge and pulsations and no pain. She asked permission to go back to work the following week in California.

Our second case was a 13-year-old girl with a right tonsillar and hypopharyngeal vascular malformation. She had recurrent bleeding episodes necessitating blood transfusions in their province. I suggested our procedure which the parents and the patient consented to. We did surgery, ligating the right external carotid artery and external jugular vein and introduced the sclerosant (sclerogen) slowly until the tonsillar and hypopharyngeal malformation blanched out.

There was no abnormal fluctuation of the patient's vital signs. After closing the surgical access wound, I did tonsillectomy of the right since the bulging tonsillar malformation was obstructing the airway. There was very minimal bleeding and I was able to cauterize the remaining sclerosed malformation not included in the tonsil with ease. Two weeks post-surgery, she followed up with healed tonsillectomy wound and a disappearing malformation. She, however, had gastritis because of her co-amoxiclav antibiotic and her inability to eat well because of pain in swallowing. She eventually recovered from her gastric problems. At one-month follow-up, there was no trace of the malformation on visual examination. Looking back, doing tonsillectomy in an AVM would have been very bloody without sclerotherapy.

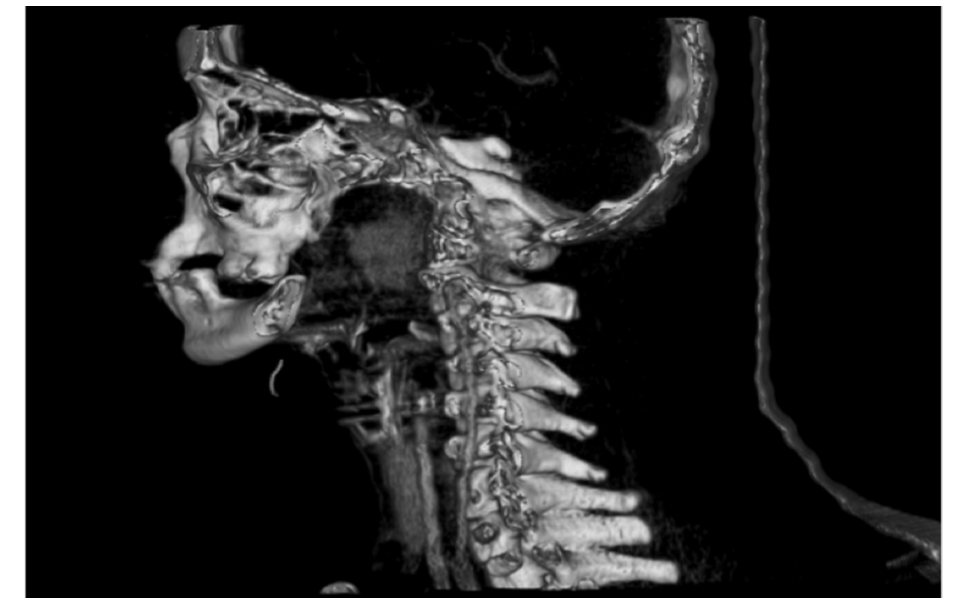

Figure 1. CT-angiography of 35-year-old woman with nasopharyngeal AVM (Case 3). It shows a nasopharyngeal vascular malformation with two arterial blood supplies-- one from the left external carotid artery as the main blood supply and another from a branch of the left internal carotid artery.

Our third case was a nasopharyngeal AV malformation in a 35-yearold woman. She had episodes of severe bleeding requiring emergency tracheostomy, oral packing and blood transfusions. CT-angiography revealed two feeding vessels, one from the left external carotid artery and a minor one from the internal carotid artery. We decided to sclerose the left external carotid artery and see what happened to the internal carotid artery branch that could not be accessed. Since CT angiography did not identify the venous drainage, we introduced the sclerosant (sclerogen) very slowly, stopping when the pulse rate started to drop below 60 beats per minute and resuming slow injection when the pulse rate was normal. Oxygen saturation was noted to be stable at 98 to $100 \%$. We stopped when the AVM blanched out, injecting 15 $\mathrm{ml}$ of sclerosant. In two weeks time, the AVM shrank except for a $1 \times 1$ $\mathrm{cm}$ bulge at the left posterior nasopharynx that was supplied by the internal carotid artery branch that could not be sclerosed at the time of surgery. The patient was decannulated from tracheostomy and was able to resume normal diet and activity.

She is on regular follow-up and is being maintained with propranolol $40 \mathrm{mg}$ once a day hoping that it may work as it does in hemangioma. One year postoperatively, the bulge has not grown nor disappeared. Looking back, had we done surgery as suggested by colleagues, we could have encountered massive bleeding, inability to take the AVM all out and eventual recurrence. Pondering upon the case of a second arterial blood supply of the AVM, the malformation could have recruited this second blood supply. The forward force of introduction of the sclerosant was not able to overcome the arterial pressure of the internal carotid artery feeding branch so the sclerotherapy effect stopped where the flow forces where at equilibrium. Note that in this case, we did not ligate the specific venous drainage as the CT-angiography did not identify it. 


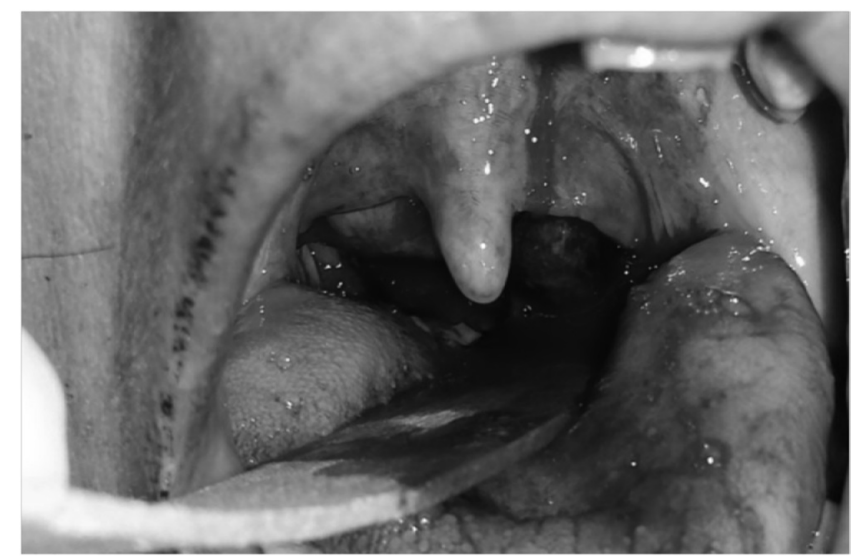

Figure 2. Transoral view of the same patient showing a dark discolored bleeding nasopharyngeal mass visible from the oropharynx. We secured the airway with tracheostomy.

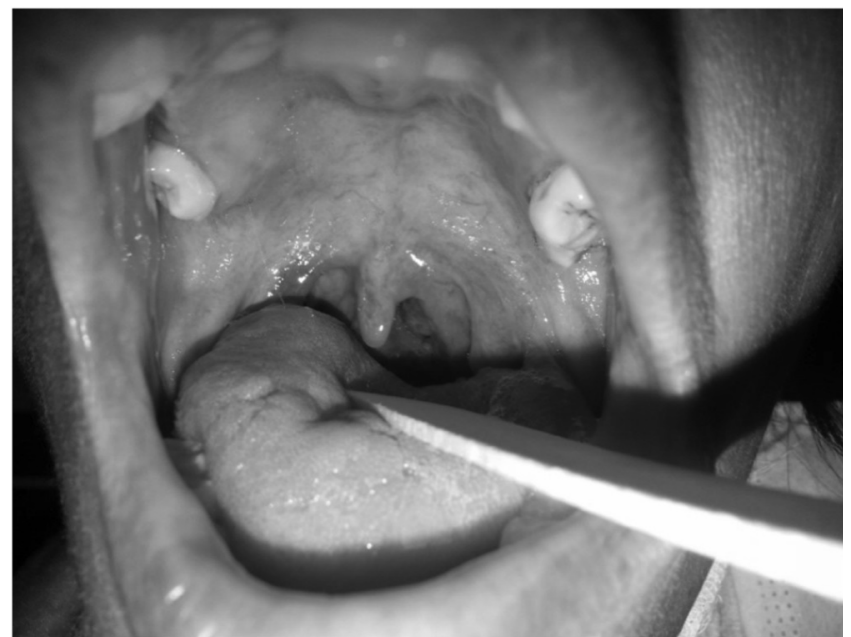

Figure 3. The same patient 2 weeks post sclerotherapy of the nasopharyngeal AVM. Note the disappearance of the bulk of the AVM except for a $1 \times 1 \mathrm{~cm}$ discolored bulge at the left posterior nasopharynx. She was weaned off tracheostomy and decannulated.

\section{DISCUSSION}

Managing AVMs that are diffuse and infiltrative can be very difficult. Surgical extirpation of all the nidus may not be possible and will surely lead to recurrences. Besides, malformations located in functionally strategic areas may present with structural deformities and functional disturbances when they are damaged by surgery. Small AVMs can be resected with high rates of success and no recurrences. In one series, all 16 patients with surgically accessible, localized, non-infiltrating AVMs who underwent angio-embolization with subsequent surgical excision demonstrated no evidence of recurrence on angiography during follow-up averaging 24.3 months. ${ }^{9}$

In low flow venous malformations, sclerotherapy administered by trans-arterial, trans-venous or direct puncture injection without embolization or feeding vessel ligation has a 64 to $96 \%$ response rate, defined as improvement in symptoms or reduction of the lesion and not necessarily cure. ${ }^{3}$

The cure rate for small malformations was $69 \%$ with excision only and $62 \%$ for extensive lesions with combined embolization and resection. At 6 years average follow-up, cure rate was $75 \%$ for stage $1 ; 67 \%$ for stage $2 ;$ and $48 \%$ for stage 3 malformations. The outcomes were not significantly affected by age at treatment, Schobinger stage or treatment method. ${ }^{11}$

Embolo-sclerotherapy is a new therapeutic modality for surgically inaccessible lesions like diffuse and infiltrating AVMs. ${ }^{4}$ This procedure is done repeatedly since the embolus recanalizes and the sclerosant is injected distal to the embolus. This method is reserved as an adjunct to subsequent surgical resection. ${ }^{4}$

Our immediate results for ligation of feeding artery or draining vein before sclerotherapy were dramatic without functional or anatomic compromise. With sclerogen whose components exist in the body naturally, we found no significant complications in our three cases. This technique could be ideal for diffuse and infiltrating AVMs. It is more effective if the feeding artery and the draining vein are identified and ligated so that the sclerosant can be pushed to all branches of the AVM. With a "no-flow or a slow-flow" AVM, we are able to prolong the contact of the sclerosant with the vascular endothelium thereby increasing the success rate of totally eliminating the nidus of the vascular malformation.

More studies and experience are needed to prove the durability of this technique. Are we able to eliminate all the nidus of the AVM if we are able to ligate all feeding arteries and draining veins before sclerotherapy? Our center is not so equipped so more technically advanced institutions dealing with vascular tumors and malformations can validate the efficacy of this technique. After all, it might not be bane to ligate AVM feeding and draining vessels if we are able to destroy the entire nidus of the AVM by whatever means like sclerotherapy in this case.

\section{ACKNOWLEDGEMENTS}

I wish to acknowledge my anesthesiologist Dr. Julius Apostol who encouraged me to try new things and promised to research on management of possible egress of sclerosants in the systemic circulation as he puts my patients to sleep. My residents at the Department of Otolaryngology-Head and Neck Surgery, Baguio General Hospital did the pre- and post-operative patient care while I was away, and the photography: Dr. Carlo Pagalilauan, Chief Resident and photographer, Dr. Sherwin Valdez, Dr. Beverly Carbonel, Dr. Jeff Peckley and Dr. Wingleaf Yu who are my assistants. Thank you.

\section{REFERENCES}

1. Richter GT, Friedman AB. Hemangiomas and vascular malformations: current theory and management. Int J Pediatr. 2012;2012:645678. doi: 10.1155/2012/645678. Epub 2012 May 7.

2. Marler JJ, Mulliken JB. Current management of hemangiomas and vascular malformations. Clin Plast Surg. 2005 Jan; 32(1): 99-116.

3. Hyodoh H, Hyodoh K. Arteriovenous Malformation: Ethanolamine Oleate sclerotherapy Dept. of Radiology, Sapporo Medical University, Japan UDK 616-131-14-007-085-089.819.

4. Wakita S, Harii K, Furuya F, Ooura N. Percutaneous monoethanoleamineolate injection sclerotheraphy for hemangiomas. Reconstructive Aesthetic Surgery. Amsterdam, New York: Kugler Publications 1995. P. 289

5. Corti P, Young S, Chen CY, Patrick MJ, Rochon ER, Pekkan K, et al. Interaction between alk1 and blood flow in the development of arteriovenous malformations. Development. $2011 \mathrm{Apr}$ 138(8): 1573-1582.

6. Kim H, Weinsheimer S, Pawlikowska L, Su H, Young WL. Brain arteriovenous malformation pathogenesis: a response-to-injury paradigm. Acta Neurochir Suppl. 2011; 111: 83-92.

7. Duyca LJ, Fan CY, Coviello-Malle JM, Buckmiller L, Suen JY. Progesterone receptors identified in vascular malformations of the head and neck. Otolaryngol Head Neck Surg. 2009 Oct; 141(4): 491-495.

8. Cronenwett JL, Johnston KW. Ruthersford's Vascular Surgery. Philadelphia: Saunders Elsevier. 2014 Apr. [cited 2014 May 28] Available from: http//www.elsevier.com.rutherfords-vascular surgery./cronenwett/9.

9. Sloan SB, Wilk R, Butler DF, Eisen D, Quirk CM, James WD, et al. Oral hemangioma treatment and management. c1994-2014 [updated 2014 Jan 30] [cited 2014 May 28] Available from http:// emedicine.medscape.com/article/1080571-treatment.

10.Speer AL, Panossian A, Arkader A, Stanley P, Anselmo DM, Stillmant RM, et al. Vascular surgery for arteriovenous malformation. Medscape Medpulse. 2012;8 [updated 2012 Nov 8]. [cited 2014 May 28] Available from: E medicine.medscape.com/article/459927.

11. Kohout MP, Hanen M, Pribaz JJ, Mulliken JB. Arteriovenous malformation of the head and neck: natural history and management. Plast Reconstr Surg. 1998 Sept; 102(3) 643-54.

12. Yakes WF, Haas DK, Parker SH, Gibson MD, Hopper KD, Mulligan JS, et al. Symptomatic vascular malformations: ethanol embolotherapy. Radiology. 1989 Mar; 170(3 Pt 2):1059-1066. 